التصنيف المظهري العددي لسبعة أجناس من عائلة خنافس الجلود في العراق باستخذام التحليل العنقودي

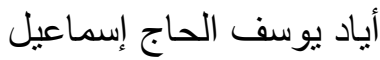

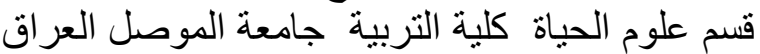

aeadismail@yahoo.com البريد الالكتروني العياء

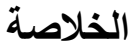

تضمن البحث التصنيف العددي للأجناس الحشرية السبعة التابعة لعائلة خنافس الجلود من رتبة غمدية

Anthrenus ,Thylodrias, Dermestes, Attagenus, Trogoderma ,Phradonoma

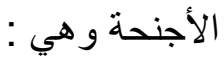

,Globicornis ,(Family Dermestidae, Order Coleoptera)

في العراق وذللك بالاعتماد على الصفات المظهرية التي تم تحويلها إلى شفرة مناسبة للتحليل العددي عبر برنامج التحليل الإحصائي الجاهز

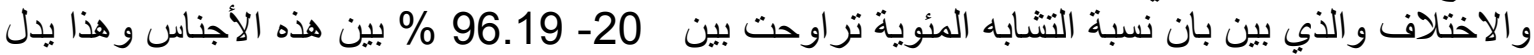

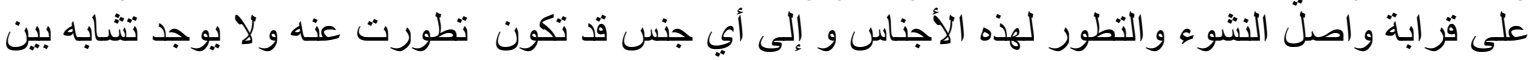

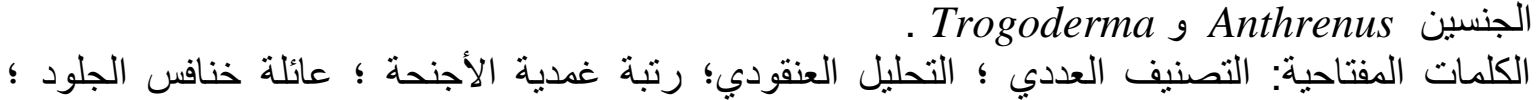
العراق.

تاريخ تسلم البحث 17 / 5 / 2013 وقبوله 7 / 2013 /

Hierarchical Classification or عرف التصنيف العددي أو التصنيف التدريجي أو التسلسلي

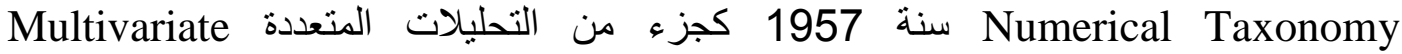
Quantitative وبشكل منوازي مع ظهور الحاسوب حيث مكن من التقارب الكمي مني (1957،Sneath)

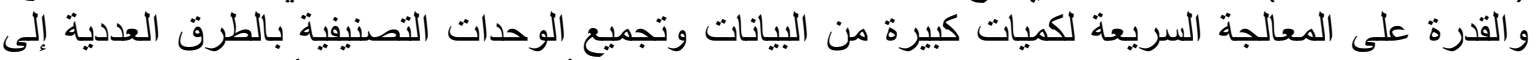

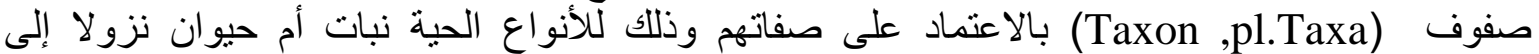

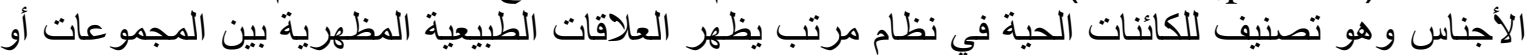

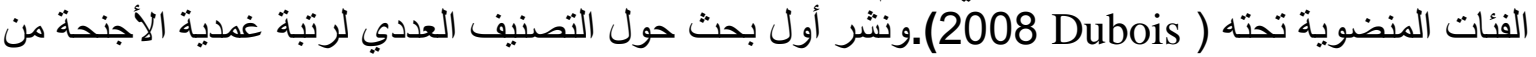

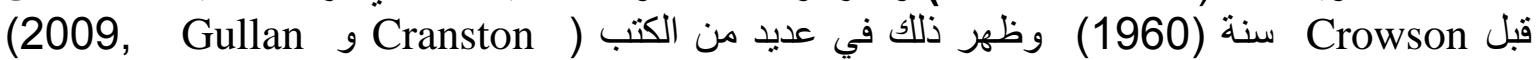
و المو اقع الالكترونية وذلك لإظهار القرابة وتحديد خطوط النشوء والارنقاء ) المحتملة لتلك الأنواع ، درس Clustral Phylogenic Trees التحليل ألنشوئي لعائلة خنافس الجلود من رتبة غمدية الأجنحة معتمدا على الصفات المات المظهرية لمرحلة اليرقة

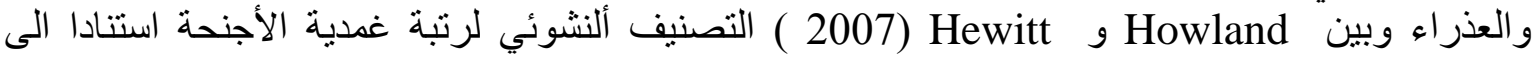

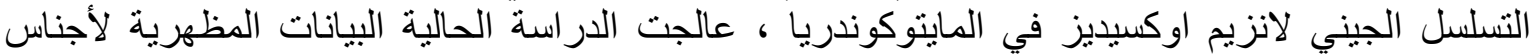

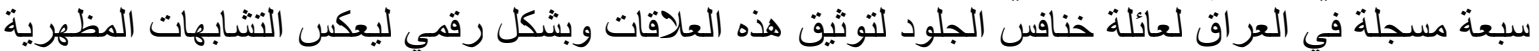

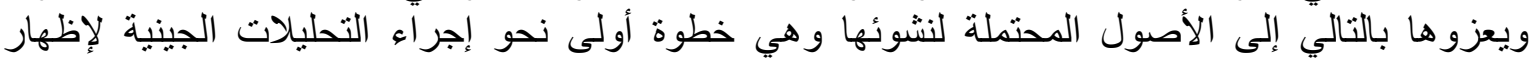
العلاقات التطورية بين هذه الأجناس ، وكذللك تمهيدا لبناء قاعدة بيانات للتصني التصنيف الآلي لهذه العائلة.

\section{مواد البحث وطرائقه}

بالاستتاد إلى المفتاح التصنيفي الذي أورده مولود (1985) للصفات المظهرية لأجناس عائلة خنافس الجلود

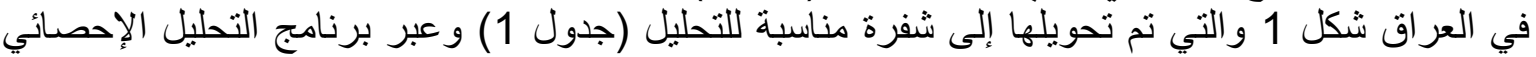
الجاهز SPSS-17 ، قورنت الصفات بإعطاء كلا منها وزنا متساويا ثم تم إنشاء مصفوفة للصفئ للصفات 2007 ) (جدول (ج) Anonymous) 


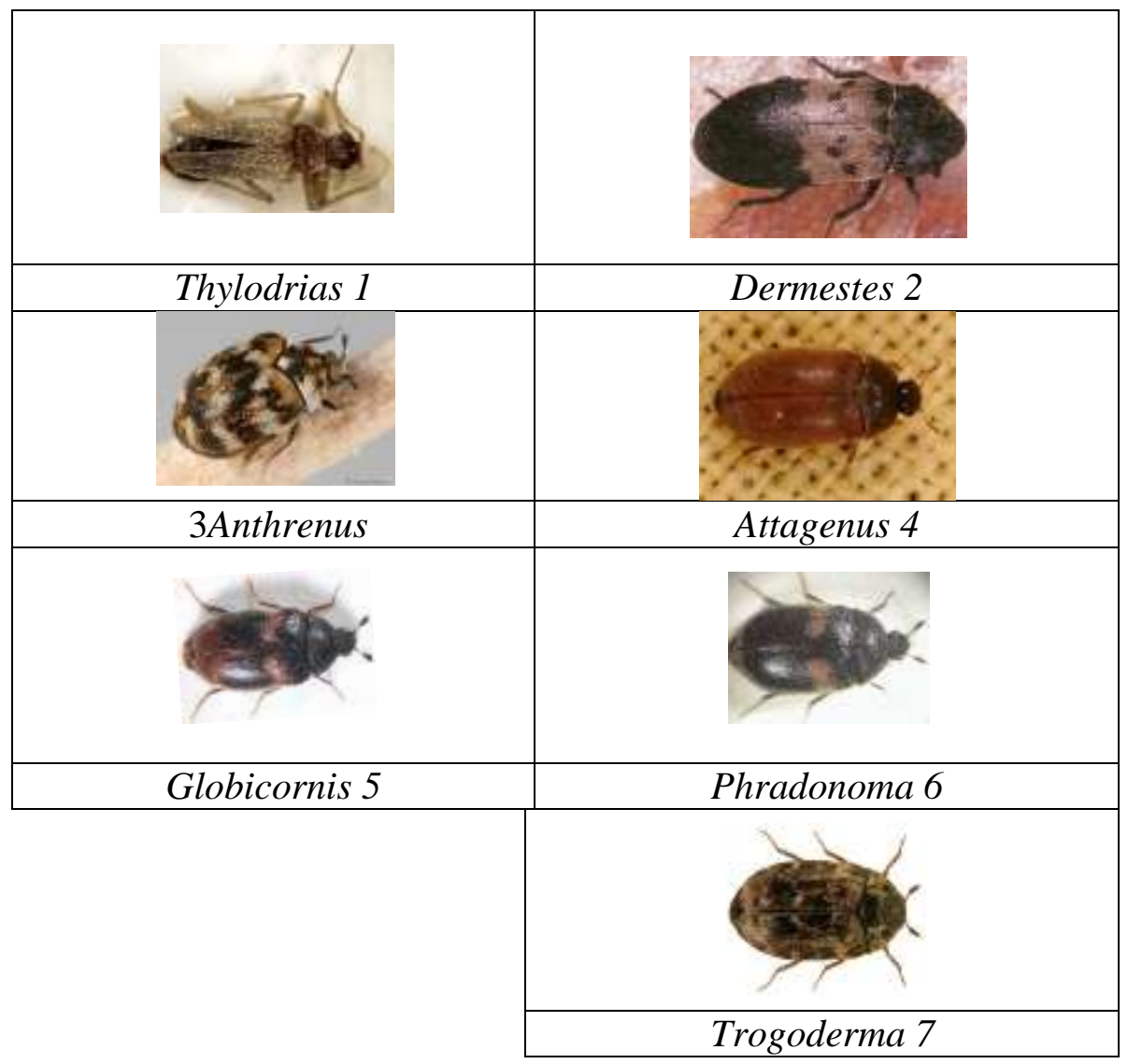

الأرقام المجاورة للأسماء تدل على تسلسل ذللك الجنس في الجدول (2).

${ }^{*}$ Numbers behind names indicated sequence of this genus in Tab 2.

$$
\text { الثكل (1) : صور أجناس عائلة خنافس الجلود في العراق. }
$$

Fig (1): Photos of the family Dermestidae genus in Iraq.

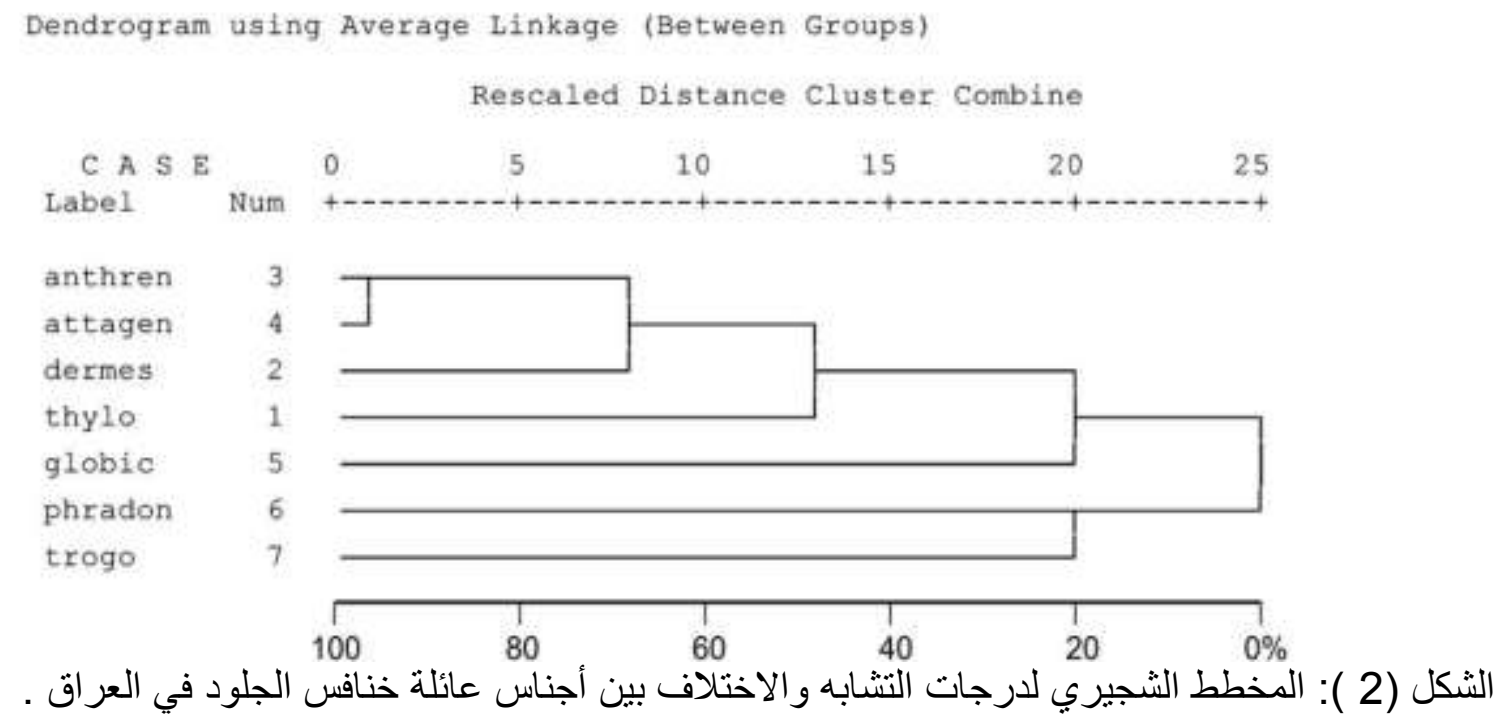

Figure(2): Dendrogram between genus of Dermestidae in Iraq .

وقائع المؤتمر الدولي الثاني لعلوم وقاية النبات 19-20 تشرين الثاني 2013 كلية الزراعة والغابات /جامعة الموصل 
جدول(1) :الصفات المظهرية المنتخبة لرسم الأشكال متعددة الأضلاع في التصنيف العددي لأجناس عائلة خنافس الجلود في العراق

Table(1):Morphological characteristic selected to description multisided shape of numerical taxonomy of Dermestidae in Iraq

\begin{tabular}{|c|c|c|c|}
\hline الثفرة Code & Specialties التفصيلات & الصفات Characteristics & Series \\
\hline $\begin{array}{l}2 \\
3\end{array}$ & 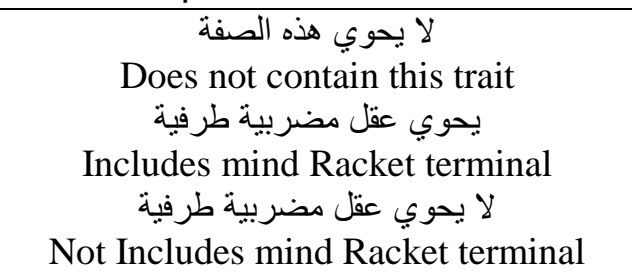 & $\begin{array}{c}\text { نوع ألنأمس } \\
\text { Type the antenna }\end{array}$ & 1 \\
\hline $\begin{array}{l}2 \\
3\end{array}$ & $\begin{array}{c}\text { لا يحوي هذه الصفة } \\
\text { Does not contain this trait } \\
\text { يحوي عين وسطية الر أس خالي من العين الوسطية } \\
\text { Includes median eye } \\
\text { Head-free median eye }\end{array}$ & $\begin{array}{c}\text { احتواء الر أس على عين وسطية } \\
\text { Contain the head median eye }\end{array}$ & 2 \\
\hline $\begin{array}{l}1 \\
2\end{array}$ & 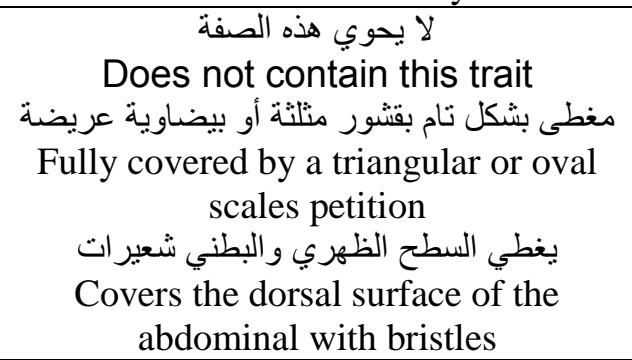 & $\begin{array}{l}\text { تغطية السطح الظهري و البطني } \\
\text { Cover the surface of the } \\
\text { dorsal and ventral }\end{array}$ & 3 \\
\hline 1 & 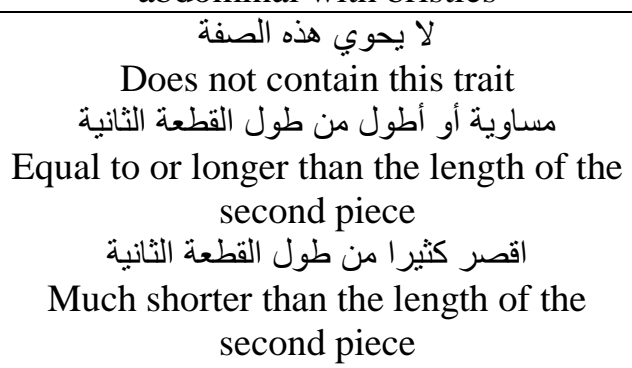 & $\begin{array}{c}\text { طول القطعة الأولى لرسغ الرجلية } \\
\text { The length of the first piece } \\
\text { of the hand-leg }\end{array}$ & 4 \\
\hline 1 & $\begin{array}{c}\text { لا يحوي هذه الصفة } \\
\text { Does not contain this trait } \\
\text { من } 11 \text { عقلة } 11 \text { pieces } \\
\text { From } 11 \text { منلة } 10-9 \text { عقلة } \\
\text { From 9-10 pieces }\end{array}$ & $\begin{array}{c}\text { تكوين أللأمس في كلا الجنسين } \\
\text { Antenna configuration in } \\
\text { both sexes }\end{array}$ & 5 \\
\hline 3 & 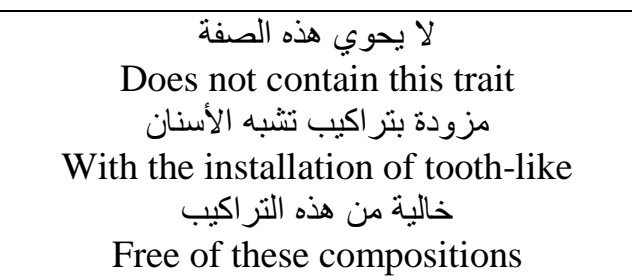 & $\begin{array}{c}\text { الحافة الخارجية أو الظهرية لساقة الأمامية } \\
\text { The outer or the dorsal edge } \\
\text { tibia of the front leg }\end{array}$ & 6 \\
\hline
\end{tabular}

وقائع المؤتمر الدولي الثاني لعلوم وقاية النبات 19-20 تشرين الثاني 2013 كلية الزراعة والغابات /جامعة الموصل 
جدول(2): مصفوفة الصفات لرسم الأشكال عديدة الأضلاع في التصنيف العددي لأجناس عائلة خنافس

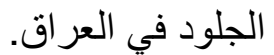

Table(2):Description multisided shape matrix of the morphological characteristic Dermestidae genus in Iraq

\begin{tabular}{|c|c|c|c|c|c|c|c|}
\hline \multicolumn{6}{|c|}{ الصفات Characteristics } & \multirow{2}{*}{ الأجناس } & \multirow{2}{*}{ Series } \\
\hline \multicolumn{2}{|c|}{5} & & & 2 & 1 & & \\
\hline 1 & 1 & 1 & 1 & **1 & 3 & Thylodrias & 1 \\
\hline 1 & 1 & 1 & 1 & 3 & 2 & Dermestes & 2 \\
\hline 1 & 1 & 1 & 2 & 2 & 2 & Anthrenus & 3 \\
\hline 1 & 1 & 3 & 2 & 2 & 2 & Attagenus & 4 \\
\hline 1 & 3 & 2 & 2 & 2 & 2 & Globicornis & 5 \\
\hline 2 & 2 & 2 & 2 & 2 & 2 & Phradonoma & 6 \\
\hline 3 & 2 & 2 & 2 & 2 & 2 & Trogderma & 7 \\
\hline
\end{tabular}

*A value of 1 means this does not have • • • genus this status

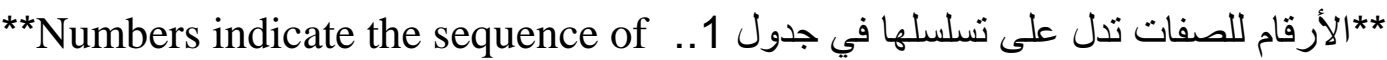
recipes in Table 1

الجدول (3): مصفوفة نسب التثابه والاختلاف في المر اتب التصنيفية لأجناس عائلة خنافس الجلود في

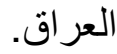

Table (3): Proximity matrix of family Dermestidae genus in Iraq .

\begin{tabular}{|c|c|c|c|c|c|c|c|}
\hline & \multicolumn{7}{|c|}{ Correlation between vectors of values } \\
\cline { 2 - 8 } Case & $\begin{array}{c}1: \\
\text { Thylo. }\end{array}$ & $\begin{array}{c}2: \\
\text { Dermes. }\end{array}$ & $\begin{array}{c}\text { 3: } \\
\text { Anthren. }\end{array}$ & $\begin{array}{c}4: \\
\text { Attagen. }\end{array}$ & $\begin{array}{c}5: \\
\text { Globic. }\end{array}$ & $\begin{array}{c}6: \\
\text { Phradon. }\end{array}$ & $\begin{array}{c}\text { 7: } \\
\text { Trogo. }\end{array}$ \\
\hline 1:Thylo. & & $.250^{*}$ & .408 & .408 & .000 & .000 & -.250 \\
2:Dermes. & .250 & & .612 & .612 & .000 & .000 & -.375 \\
3:Anthren. & .408 & .612 & & 1.000 & .000 & .000 & -.612 \\
4:Attagen. & .408 & .612 & 1.000 & & .000 & .000 & -.612 \\
5:Globic. & .000 & .000 & .000 & .000 & & .000 & -.791 \\
6:Phradon. & .000 & .000 & .000 & .000 & .000 & & .000 \\
7:Trogo. & -.250 & -.375 & -.612 & -.612 & -.791 & .000 & \\
\hline
\end{tabular}

${ }^{*}$ This is a similarity matrix

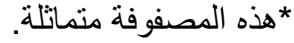

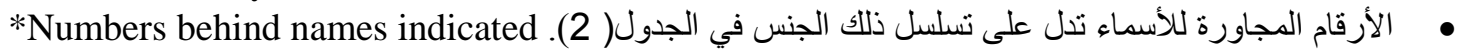
sequence of this genus in Tab2.

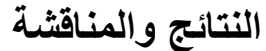

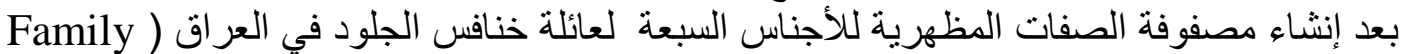
(Dermestidae : Order Coleoptera

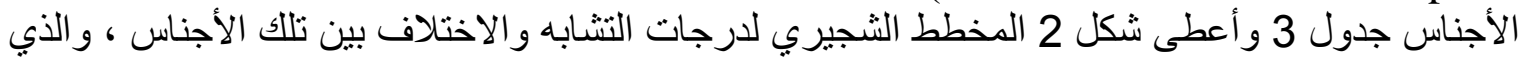

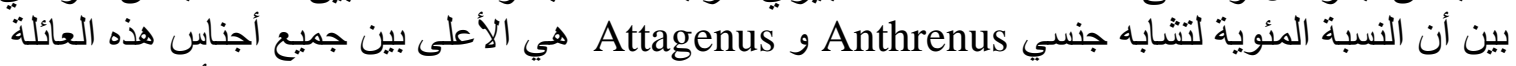

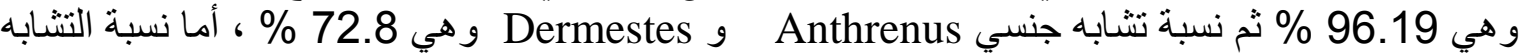

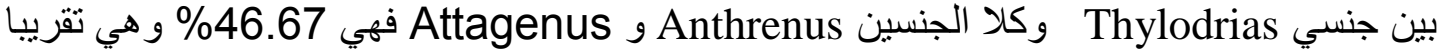
نصف المسافة بين اجناس هذه العائلة وان نسبة التشابه بين Anthrenus و و و و و والجنسين Trogoderma و Phradonoma 
** الأرقام الغامقة تدل على اسم ذلك الجنس في الجدول (1) و الأرقام الصغيرة الفاتحة تدل على الصفات الددروسة.

** Dark figures indicate the name of that genus in Table 1 and Figures small light show traits.

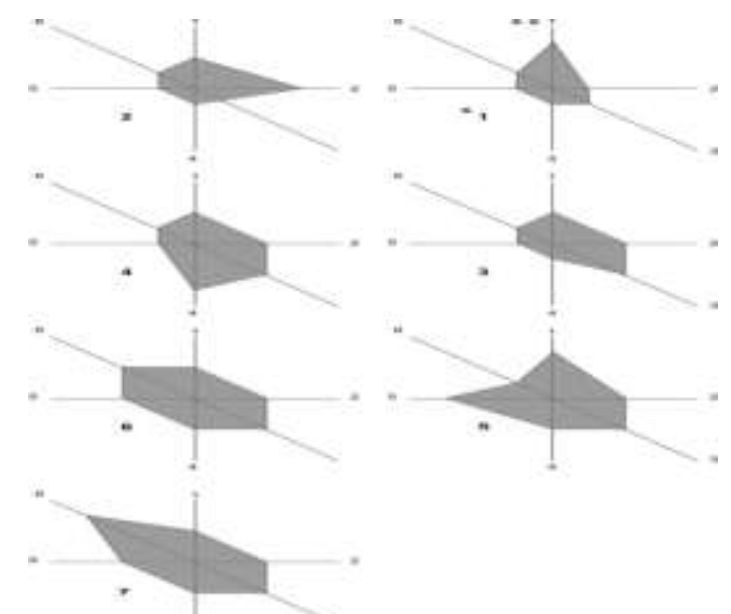

$$
\text { الثكل (3) : رسم عديد الأضلاع للصفات المظهرية لأجناس عائلة خنافس الجلود في العر اق. }
$$

Figure (3) :Multisided shape of morphological characteristics of Dermestidae genus in Iraq.

وهذا يدل على قرابة و اصل نشوء وارتقاء هذه الأجناس والى أبي جنس قد تكون تطورت عنه ، و ولا يوجد

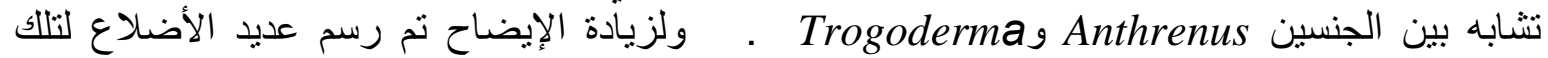

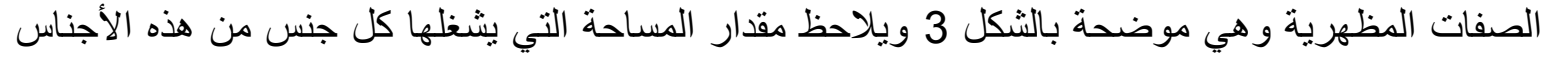

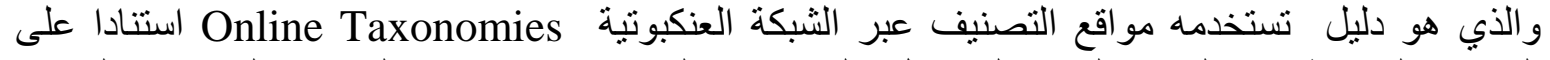

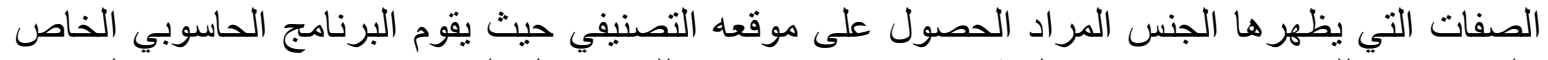
بالتصنيف وذللك باستخدام برنامج الماتثلاب Mathlab في ذلك من قبل المبرمجين متخصصين بعمل مسح

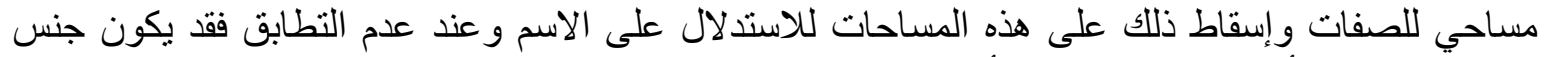
جديد لُهذه العائلة أو قد يعود إلى عائلة أخرى(

\title{
NUMERICAL TAXONOMY OF FAMILY DERMESTIDAE: COLEOPTERA IN IRAQ BY USING GLUSTERING ANALYSIS
} Aead Y. Haj Ismail

Biology Dept., Education College , Mosul University

Mosul IRAQ

E-mail:aeadismail@yahoo.com

\begin{abstract}
Morphological characteristics of insect species (Thylodaris, Dermestes, Anthernus, Globiconis, Phraonoma, Trogoderma) belong to Dermestidae :Coleoptera which are recorded in IRAQ were given equal weight for each character .A matrix was constructed for the characteristics a dendrogram and multisided shape were drown for similarity and differences between the species using Clustering Analysis Statistically Packaging SPSS-17. The results revealed that characteristics ranging 96.19-20\% . Not a single similarity was found between

وقائع المؤتمر الدولي الثاني لعلوم وقاية النبات 19-20 تشرين الثاني 2013 كلية الزراعة والغابات /جامعة الموصل
\end{abstract}


Trogoderma \& Anthrenus compared to the preceding species dealt with Cartier .

Key words: numerical taxonomy; clustering analysis; Coleoptera; Dermestidae ;IRAQ.

Received: 17 / 5 /2013 Accepted: 7 / 9 / 2013.

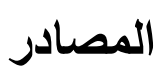

Dermestidae :Coleoptera: مولود ، نبيل عبد القادر..(1985) ـ دراسة تصنيفية لخنافس عائلة Insecta

Anonymous (2007). SPSS Statistics Base .17.0, Users Guide.616 Pages.

Cranston, Peter S. and Penny J. Gullan. (2009).Phylogeny of insects. Encyclopedia of Insects:828-882.

Crowson, R.A.(1960) .The Phylogeny of Coleoptera. Annual Review Entomology .5:111-134.

Dubois, Alain. (2008). Aphylogenetic hypotheses, taxa and nomina in zoology . Zootaxa.1950:51-86.

Houcque, David. (2005).Introduction To Mathlab For Engineering Students, Version1.2 .Northwestern University .74 Pages.

Howland, D.E. and G.M. Hewitt. (2007).Phylogeny of the Coleoptera based on mitochondrial oxidase I sequence data. Insect Molecular Biology , 4 (3):496-507.

Kiselyova, T. and J.V. McHugh .(2006). Aphylogenetic Study of Dermestidae Coleoptera Based on larva morphology . Systematic Entomology .3(3):469-50

Sneath, P.H.A.(1957). Some thoughts on bacterial classification. Journal Genetic Microbiology.17:184-200. 